\title{
Acervo Científico: 0 conhecimento sem fronteiras
}

As Revistas da Acervo+ vem publicando uma série de editoriais com o intuito de estimular a leitura científica e propiciar o acesso ao conhecimento. Nesse sentido, apresentamos um índice de artigos categorizados por área do conhecimento que foram publicados nas revistas que integram a Acervo+ Index base.

Tenham todos uma boa leitura!

Acervo+, inovando na disseminação da ciência.

\section{Eixo Multiáreas}

4 de MELO TRNB, et al. Avaliação do controle das medidas de biossegurança adotadas por acadêmicos de Odontologia. Revista Eletrônica Acervo Científico, 2020; 8: e2112. [Acessar]

4 LEAL TLSL, et al. Perfil epidemiológico dos casos de sífilis congênita no Maranhão. Revista Eletrônica Acervo Científico, 2020; 8: e2936. [Acessar]

4 MACEDO GG, et al. Fraturas do fêmur em idosos: um problema de saúde pública no Brasil. Revista Eletrônica Acervo Científico, 2019; 6: e1112. [Acessar]

de ARAÚJO FO, et al. Cultivation of the microalgae chlorella pyrenoidosa using the processes of biotechnology. Revista Eletrônica Acervo Científico; 2019; 2: 121. [Acessar]

DORTAS ARF, et al. Fatores de risco associados a sepse neonatal: artigo de revisão. Revista Eletrônica Acervo Científico, 2019; 7: e1861. [Acessar]

NUNES LM, et al. Pesquisa de coliformes totais e termotolerantes no rio Salgadinho no município de Juazeiro do Norte, CE. Revista Eletrônica Acervo Científico, 2019; 7: e2243. [Acessar]

SILVA AA, et al. Morbimortalidade hospitalar por câncer colorretal no Brasil, no período de 2008 a 2016. Revista Eletrônica Acervo Científico, 2019; 5: e939. [Acessar]

GODOI J, et al. Perda precoce do primeiro molar permanente. Revista Eletrônica Acervo Científico, 2019; 4: e729. [Acessar]

GONÇALVES MAF. A dislexia no ensino fundamental. Revista Eletrônica Acervo Científico, 2019; 3: e648. [Acessar]

MIRANDA TS, et al. Disparidades em saúde da população LGBTQIA+: a atuação médica frente a este cenário. Revista Eletrônica Acervo Científico, 2020; 13: e4872. [Acessar]

BATISTA L, MATUMOTO S. Validação de roteiro de sistematização da assistência de enfermagem à gestante na atenção primária. Revista Eletrônica Acervo Científico, 2019; 7: e1889. [Acessar]

RIBEIRO LB, et al. Oficina de arte visual baseada na obra clássica" 0 Pequeno Príncipe" voltada a jovens autistas e neurotípicos com o auxílio das neurociências. Revista Eletrônica Acervo Científico, 2019; 7: e2177. [Acessar]

COSTA AR, et al. Pancreatite crônica-fisiopatologia e tratamento: uma revisão de literatura. Revista Eletrônica Acervo Científico, 2019; 4: e779. [Acessar]

TIBONI F, et al. Revisão bibliográfica sobre regeneração óssea guiada em associação a implantes odontológicos. Revista Eletrônica Acervo Científico, 2019; 3: e913. [Acessar] 
de AZEVEDO SILVA R, et al. Coinfecção de hepatites virais e HIV na região norte do Brasil. Revista Eletrônica Acervo Científico, 2019; 4: e498. [Acessar]

de ARAÚJO FO, et al. Identification of the lipids of the microalgae Chlorella pyrenoidosa, after obtaining this through use of biotechnology. Revista Eletrônica Acervo Científico, 2019; 3: e122. [Acessar]

REIS SC. Uma revisão bibliográfica sobre aterosclerose. Revista Eletrônica Acervo Científico, 2019; 3: e444. [Acessar]

CAZAROTTI MLB, et al. Psicotrópicos: Prescrições Médicas Dispensados em uma Drogaria no Município de Santa Inés-MA. Revista Eletrônica Acervo Científico, 2019; 2: e326. [Acessar]

de SOUZA RSM, et al. A infecção por coronavírus e a cardiomiopatia de Takotsubo na era da pandemia do SARS-CoV 2. Revista Eletrônica Acervo Científico, 2021; 25: e7368. [Acessar]

BALDOW CC, et al. Infecção pelo SARS-CoV-2 na gestação: revisão de literatura. Revista Eletrônica Acervo Científico, 2021; 25: e7249. [Acessar]

\section{Eixo Saúde}

PARAGUASSU ÉC, et al. Qualidade de vida e satisfação em usuários de prótese total no estado do Amapá, Brasil. Revista Eletrônica Acervo Saúde, 2019; sup27: e876. [Acessar]

RAMOS FP, et al. Fatores associados à depressão em idoso. Revista Eletrônica Acervo Saúde, 2019; sup19: e239. [Acessar]

PRADO $A D$, et al. A saúde mental dos profissionais de saúde frente à pandemia do COVID-19: uma revisão integrativa. Revista Eletrônica Acervo Saúde, 2020; esp46: e4128. [Acessar]

LIMA SO, et al. Impactos no comportamento e na saúde mental de grupos vulneráveis em época de enfrentamento da infecção COVID-19: revisão narrativa. Revista Eletrônica Acervo Saúde, 2020; esp46: e4006. [Acessar]

da SILVA DA. A autoestima e o comportamento suicida em estudantes universitários: uma revisão da literatura. Revista Eletrônica Acervo Saúde, 2019; 23: e422. [Acessar]

ADAMI AAV, de LIMA DUTRA MB. Análise da eficácia do vinagre como sanitizante na alface (Lactuta sativa, L.). Revista Eletrônica Acervo Saúde, 2011; 3: 134-144. [Acessar]

GARCIA DP, DUARTE DA. Perfil epidemiológico de surtos de doenças transmitidas por alimentos ocorridos no Brasil. Revista Eletrônica Acervo Saúde, 2014; 6(1): 545-554. [Acessar]

MARÇAL JA, SOUZA LT. A prevenção do câncer de colo de útero realizada pelo enfermeiro na Estratégia Saúde da Família: Revisão integrativa da literatura. Revista Eletrônica Acervo Saúde, 2013; 5(2): 474-489. [Acessar]

PORTUGAL JKA, et al. Percepção do impacto emocional da equipe de enfermagem diante da pandemia de COVID-19: relato de experiência. Revista Eletrônica Acervo Saúde, 2020; esp46: e3794. [Acessar]

CUNHA DV, et al. Perfil Epidemiológico da Hanseníase no Município de Castanhal-Pará no período de 2014 a 2017. Revista Eletrônica Acervo Saúde, 2019; 11(15): e858-e858. [Acessar] 
da SILVA JV, et al. Capacidades de autocuidado e sua relação com os fatores condicionantes básicos: um estudo em Unidades Básicas de Saúde. Revista Eletrônica Acervo Saúde, 2012; 4(1): 185-199. [Acessar]

BARBOSA LU, et al. A educação sexual nos documentos das políticas de educação e suas ressignificações. Revista Eletrônica Acervo Saúde, 2019; 11(10): e772. [Acessar]

TEIXEIRA-LOYOLA ABA, et al. Análise Microbiológica de especiarias comercializadas em Pouso Alegre, Minas Gerais. Revista Eletrônica Acervo Saúde, 2014; 6(1): 515-529. [Acessar]

dos SANTOS DS, et al. Transição nutricional na adolescência: uma abordagem dos últimos 10 anos. Revista Eletrônica Acervo Saúde, 2019, sup20: e477. [Acessar]

de ALMEIDA JR, et al. O enfermeiro frente às práticas integrativas e complementares em saúde na estratégia de saúde da família. Revista Eletrônica Acervo Saúde, 2019; Sup18: e77. [Acessar]

da COSTA BARBOSA C, et al. Qualidade microbiológica da água consumida em bebedouros de uma unidade hospitalar no Sul de Minas. Revista Eletrônica Acervo Saúde; 2012; 4(1): 200211. [Acessar]

de ALMEIDA JUNIOR S, et al. Bases pedagógicas em curso profissionalizante de Farmácia e Laboratório Clínico como apoio na construção profissional do indivíduo. Revista Eletrônica Acervo Saúde, 2019; sup25: e649. [Acessar]

dos SANTOS PORTO TNR, et al. Prevalência do excesso de peso e fatores de risco para obesidade em adultos. Revista Eletrônica Acervo Saúde, 2019; sup22: e308. [Acessar]

MACHADO APMC, et al. Avaliação da adesão ao tratamento de pacientes com diabetes mellitus e seus fatores associados. Revista Eletrônica Acervo Saúde, 2019; sup19: e565. [Acessar]

GARCIA IM, et al. Percepção do discente de enfermagem na construção do seu conhecimento no contexto da metodologia ativa. Revista Eletrônica Acervo Saúde, 2019; 11(2): e127. [Acessar]

\section{Eixo Enfermagem}

dos SANTOS AA, et al. O papel do enfermeiro na prevenção do desmame precoce. Revista Eletrônica Acervo Enfermagem, 2020; 2: e2232. [Acessar]

da SILVA MR, et al. Infecção de trato urinário associada ao cateterismo vesical de demora na população idosa: classificações de enfermagem. Revista Eletrônica Acervo Enfermagem, 2020, 3: e3540. [Acessar]

de CASTRO JM, et al. Perfil de nascimentos no estado de Minas Gerais no período de 2013 a 2017. Revista Eletrônica Acervo Enfermagem, 2020; 2: e2354. [Acessar]

SEBASTIÃO GC, et al. Relato de experiência sobre o uso de celulares e adornos em uma unidade de terapia intensiva. Revista Eletrônica Acervo Enfermagem, 2019; 1: e2009. [Acessar]

PINHEIRO BM, et al. Fatores que influenciam o desmame precoce do aleitamento materno: uma revisão integrativa. Revista Eletrônica Acervo Enfermagem, 2021; 11: e7227. [Acessar] 
BARBOSA IEB, et al. Procedimentos e técnicas de enfermagem realizadas durante a pandemia de COVID-19. Revista Eletrônica Acervo Enfermagem, 2021; 9: e6016. [Acessar]

BEM FERREIRA, et al. Adesão dos profissionais de enfermagem as metas de segurança da OMS: uma revisão de literatura. Revista Eletrônica Acervo Enfermagem, 2021; 8: e5967. [Acessar]

de CASTRO RR, et al. Caracterização das ocorrências do serviço de Atendimento Móvel de Urgência-SAMU. Revista Eletrônica Acervo Enfermagem, 2020; 7: e5625. [Acessar]

de JESUS CARDOSO AO, et al. A prática de enfermagem frente aos pacientes portadores de esquizofrenia. Revista Eletrônica Acervo Enfermagem, 2020; 5: e5118. [Acessar]

RAMOS LS, et al. A humanização da atenção básica a saúde brasileira no atendimento de travestis e transsexuais: uma revisão narrativa. Revista Eletrônica Acervo Saúde, 2020; e2770. [Acessar]

de OLIVEIRA DN, et al. Desfechos obstétricos em gestações tardias no estado de Minas Gerais. Revista Eletrônica Acervo Enfermagem, 2020; 3: e2555. [Acessar]

ALMEIDA RCAA, et al. Cobertura vacinal ANTI-HPV e motivos de não vacinação. Revista Eletrônica Acervo Enfermagem, 2020; 2: e2600. [Acessar]

SILVA PDM, et al. O impacto da doença de Alzheimer na vida do cuidador. Revista Eletrônica Acervo Enfermagem, 2020; 2: e2353. [Acessar]

do NASCIMENTO DKL, et al. Atuação da enfermagem no atendimento as urgências e emergências de pacientes vítimas de queimadura: uma análise conceitual. Revista Eletrônica Acervo Enfermagem, 2019; 1: e2122. [Acessar]

CORTES HM, et al. Vivências de mulheres transgêneras de um município do recôncavo da Bahia. Revista Eletrônica Acervo Enfermagem, 2019; 1: e1871. [Acessar]

DUARTE DA. Bronquite e seus problemas relacionados: Uma revisão bibliográfica. Revista Eletrônica Acervo Enfermagem, 2019; 1: 002. [Acessar]

DUARTE DA. Benefícios da amamentação. Revista Eletrônica Acervo Enfermagem, 2019; 1: 001 001. [Acessar]

de FREITAS JC, et al. Eficácia dos métodos não farmacológicos para alívio da dor no trabalho de parto natural: uma revisão integrativa. Revista Eletrônica Acervo Enfermagem, 2021; 12 : e7650. [Acessar]

de SOUZA NASCIMENTO $T$, et al. Processo de trabalho em um Centro de Atenção Psicossocial: um relato de experiência. Revista Eletrônica Acervo Enfermagem, 2021; 11: e7755. [Acessar]

de OLIVEIRA LP, et al. Infecção urinária na gestação e as repercussões ao recém-nascido: uma revisão integrativa. Revista Eletrônica Acervo Enfermagem, 2021; 11: e7612. [Acessar]

\section{Eixo Odontologia}

de AQUINO TS, et al. Laserterapia de baixa potência no tratamento de parestesia oral-uma revisão sistematizada. Revista Eletrônica Acervo Odontológico, 2020; 1: e3753-e3753. [Acessar] 
de SÁ OLIVEIRA G, et al. Associação entre a odontologia estética e autoestima. Revista Eletrônica Acervo Odontológico, 2020; 1: e3892. [Acessar]

de OLIVEIRA ROCHA A, et al. A utilização da laserterapia para o controle da hipersensibilidade dentinária: uma revisão sistematizada da literatura. Revista Eletrônica Acervo Odontológico, 2020; 2: e3907. [Acessar]

CANALI LGM, et al. Comprometimento de papila parotídea em lesão de hiperplasia fibrosa inflamatória: relato de caso. Revista Eletrônica Acervo Odontológico, 2020; 1: e4179. [Acessar]

OLIVEIRA MMS, et al. Aspectos psicossociais relacionados ao paciente desdentado: Uma revisão da literatura. Revista Eletrônica Acervo Odontológico, 2020; 1: e2477. [Acessar]

da CONCEIÇÃO MONTEIRO R, CASTRO ALS. Educação continuada em saúde bucal para professores da educação infantil: contexto atual e importância para a odontologia preventiva. Revista Eletrônica Acervo Odontológico, 2021; 3: e6082. [Acessar]

TRINDADE GD, et al. Efeitos de extrato em gel de chenopodium ambrosioides I.(mastruz) no tratamento de lesões ósseas de ratas osteoporóticas. Revista Eletrônica Acervo Odontológico, 2021; 3: e6260. [Acessar]

MONTEIRO GG, et al. Resinas bulkfill: propriedades e aplicabilidades. Revista Eletrônica Acervo Odontológico, 2021; 3: e6128. [Acessar]

da SILVA LIMA A, et al. O papel do 10-MDP no processo da adesão. Revista Eletrônica Acervo Odontológico, 2021; 3: e5759. [Acessar]

de SOUZA CM, et al. Manifestações clínicas bucais da paracoccidioidomicose: um relato de caso. Revista Eletrônica Acervo Odontológico, 2021; 3: e5893. [Acessar]

CARVALHO TA, et al. Manchas extrínsecas negras em dentes decíduos e permanentes: revisão da literatura. Revista Eletrônica Acervo Odontológico, 2020; 2: e5915. [Acessar]

BATISTA SG, et al. Levantamento de seio maxilar bilateral por duas técnicas diferentes com concomitante instalação de implantes: relato de caso. Revista Eletrônica Acervo Odontológico, 2020; 2: e5878. [Acessar]

da SILVA SOUSA Z, et al. Selamento de dentina cariada: uma alternativa minimamente invasiva. Revista Eletrônica Acervo Odontológico, 2020; 2: e5743. [Acessar]

PEREIRA RL, et al. Remoção de retentor intrarradicular com auxílio de microscopia: relato de caso. Revista Eletrônica Acervo Odontológico, 2020; 2: e5170. [Acessar]

SARMENTO MGS, et al. Desafios da educação em saúde bucal na adolescência. Revista Eletrônica Acervo Odontológico, 2020; 2: e4249. [Acessar]

de MELO TRNB, SOUZA GR. Avaliação da distância intercaninos e sua correlação com as más oclusões. Revista Eletrônica Acervo Odontológico, 2020; 1: e3937. [Acessar]

de ANDRADE BATISTA LCS, et al. A sífilis e suas manifestações bucais: relato de caso clínico. Revista Eletrônica Acervo Odontológico, 2020; 1: e3446. [Acessar]

FONSECA RCA, da SILVA FB. Vedamento de comunicação buco-sinusal com prótese obturadora palatina: relato de caso. Revista Eletrônica Acervo Odontológico, 2020; 1: e3117. [Acessar] 
de MELO TRNB, et al. Tração reversa da maxila: relato de caso clínico. Revista Eletrônica Acervo Odontológico, 2020; 1: e3252. [Acessar]

\section{Eixo Ensino e Educação}

de SOUZA AJ. Considerações sobre o brincar: porque os brinquedos auxiliam e podem influenciar nos processos de aprendizado. Revista Acervo Educacional (online), 2020; 2: e2126. [Acessar]

4t da SILVA EA. O Ensino de Física e as Energias Renováveis. Revista Acervo Educacional (online), 2019; 1: e1309. [Acessar]

MAGALHÃES DSS, et al. Estágio curricular supervisionado: expectativas na formação profissional. Revista Acervo Educacional (online), 2021; 3: e6584. [Acessar]

VIEIRA L, et al. Relato de experiência: a construção de um curso pré-vestibular popular vinculado ao curso de medicina. Revista Acervo Educacional (online), 2021; 3: e6855. [Acessar]

PROCÓPIO ER, et al. Letrando em tempos de cibercultura: o desafio no processo de alfabetizar. Revista Acervo Educacional (online), 2021; 3: e5134. [Acessar]

de ALMEIDA TEODORO LC. A importância da educação na construção da cidadania: uma análise do Programa Bolsa Família. Revista Acervo Educacional (online), 2020; 2: e4086. [Acessar]

da SILVA RL. Relato de experiência: teoria especial da relatividade interação física-literatura em uma disciplina eletiva do ensino integral da Paraíba. Revista Acervo Educacional (online), 2020; 2: e4003. [Acessar]

PEIXOTO FJG. Questões éticas a partir do filme "O clube do imperador": uma revisão bibliográfica. Revista Acervo Educacional (online), 2020; 2: e3234. [Acessar]

TAVARES PTN, CLOUX RF. O uso das tics nas práticas de leitura e escrita na sala de aula. Revista Acervo Educacional (online), 2020; 2: e2835. [Acessar]

RIBEIRO LB, et al. Rebuscando o Português: por uma neuroplasticidade diferente. Revista Acervo Educacional (online), 2020; 2: e2494. [Acessar]

FONSECA CR, et al. Desafios dos alunos com deficiência auditiva no ensino regular. Revista Acervo Educacional (online), 2019; 1: e1883. [Acessar]

GONTIJO HL, et al. Relato de experiência: Universidades das Crianças em Minas Gerais. Revista Acervo Educacional (online), 2019; 1: e1629. [Acessar]

LIMA GJS, MOURA LT. Educador: Despertando consciências. Revista Acervo Educacional (online), 2019; 1: e1258. [Acessar]

\section{Eixo Multiáreas}

RIBEIRO DR, et al. Prevalência de diabetes mellitus e hipertensão em idosos. Revista Artigos. Com, 2020; 14: e2132. [Acessar]

MOL MAL, et al. Tabagismo e desfechos cardiovasculares entre hipertensos. Revista Artigos. Com, 2019; 12: e2566. [Acessar]

GUINHAZI NP, et al. Indicações e condutas de rastreamento de pólipos intestinais: uma revisão de literatura. Revista Artigos. Com, 2019; 1: e158. [Acessar] 
GAMA BTB, et al. Seletividade alimentar em crianças com Transtorno do Espectro Autista (TEA): uma revisão narrativa da literatura. Revista Artigos. Com, 2020; 17: e3916. [Acessar]

COTRIM TS, et al. Avaliação do pré-natal de usuárias cadastradas em uma Estratégia Saúde da Família de uma cidade do Pará. Revista Artigos. Com, 2020; 17: e3466. [Acessar]

VALARISTINO JM, et al. Atuação do enfermeiro na atenção domiciliar: revisão narrativa. Revista Artigos. Com, 2019; 12: e2567. [Acessar]

COSTA AM. A escalada do efeito Dunning-Krueger na desconstrução do conhecimento científico. Revista Artigos. Com, 2019; 11: e2267. [Acessar]

RIBEIRO DR, et al. Atendimento de enfermagem na área de urgência e emergência pediátrica. Revista Artigos. Com, 2019; 10: e2130. [Acessar]

PINHEIRO LAP, da SILVA RA. A legislação penal do Feminicídio. Revista Artigos. Com, 2019; 7: e1785. [Acessar]

TORRES RA. A Educação Física na Alfabetização. Revista Artigos. Com, 2019; 7: e1743 [Acessar]

VIEIRA KLSS. Evolução tecnológica e a reestruturação do espaço. Revista Artigos. Com, 2019; 3: e1118. [Acessar]

da SILVA CR. Interdisciplinaridade: conceito, origem e prática. Revista Artigos. Com, 2019; 3: e1107. [Acessar]

LIMA JMP. A importância da sequência didática para a aprendizagem significativa da matemática. Revista Artigos. Com, 2019; 2: e829. [Acessar]

RA SANTANA, et al. Políticas públicas de meio ambiente e seus efeitos na saúde. Revista Artigos. Com, 2019; 2: e720. [Acessar]

de OLIVEIRA TF, et al. COVID-19: REVISÃO NARRATIVA. Revista Artigos. Com, 2021; 25: e4252. [Acessar]

SOUZA ACOC, et al. A influência da laserterapia de baixa potência e do ultrassom terapêutico na abertura da boca em pacientes com disfunção temporomandibular. Revista Artigos. Com, 2020; 22 : e6006. [Acessar]

da SILVA LAS, et al. Principais aplicações dos resíduos da laranja e sua importância industrial. Revista Artigos. Com, 2020; 22: e5139. [Acessar]

da SILVA JN. OBESIDADE E COVID-19: QUAIS AS EVIDÊNCIAS? Revista Artigos. Com, 2020; 1, 21: e5346. [Acessar]

CAFÉ LA, et al. A atuação do enfermeiro na saúde mental. Revista Artigos. Com, 2020; 21: e5016. [Acessar]

RODRIGUES EKB, ARRUDA SG. Mortalidade Por Causas Externas Na Região Norte Do Espírito Santo, 2010 A 2018. Revista Artigos. Com, 2020; 20: e4258. [Acessar] 